\title{
DESIGN OF AN EASY-TO-USE MOBILE AUGMENTED REALITY LEARNING SETTING BY MEANS OF A CONJECTURE MAP
}

\author{
Luca Moser \\ University of St.Gallen, Switzerland \\ St. Jakob-Strasse 21, CH-9000 St.Gallen, Switzerland
}

\begin{abstract}
Despite the positive effects of mobile augmented reality (MAR)-tools for learning, MAR-tools are not commonly used in classrooms. The scientific discourse identified a lack of concepts that guide the practical application of mobile augmented reality (MAR)-tools in education. Teachers often feel insecure when designing and applying digital learning settings and therefore need tools and concepts to support them. The present contribution outlines how to develop an easy-to-use mobile augmented reality learning setting (MARLS). Thus, a MARLS to foster artificial intelligence (AI)-literacy is developed and studied to explore what makes MARLS easy-to-use. AI-literacy serves as an exemplary topic. It appears to be suiting because MAR-technology is based on AI-systems and hence allows students to experience a positive form of human-AI-interaction first headedly. The educational design research is conducted by means of a conjecture map to enable the concurrent investigation of learning, teaching and its interdependence. Derived from the high-level conjectures, (I): AI-literacy is a set of competences, (II): a digital learning environment is crucial to foster it, (III): a good MARLS considers usability, user centeredness, conscious application, basic learning theories and cognitive load, the MARLS is developed. The MARLS and its conjecture map lay ground for the yet to follow measure and improvement of the learning design.
\end{abstract}

\section{KEYWORDS}

Marker Based Augmented Reality, Mobile Augmented Reality, AI-literacy, Conjecture Map, Education, Educational Design Research

\section{INTRODUCTION}

Mobile augmented reality (MAR)-applications are increasingly discussed in the field of education (Akçayır and Akçayır, 2017; Pedaste et al., 2020). Like other augmented reality (AR)-tools, they are designed to "supplement the reality" with virtual elements (Azuma, 1997) and create an augmented environment where physical and digital features complement each other (Davies, 2004; Milgram and Kishino, 1994). Distinct about MAR-tools is that they can be applied with any mobile device (smartphone or tablet computer). Most upper secondary students own a smartphone (Cano and Sevillano-Garcia, 2018; Suter et al., 2018), what makes MAR-tools particularly cost-effective, as no further hardware has to be acquired. Hence, MAR-tools are potentially easier to distribute on a big scale than other AR-tools (Akçayır and Akçayır, 2017). Furthermore, the most comprehensive meta-studies on the subject identified significant potential for MAR-learning settings (MARLS) to increase learner engagement, motivation, learning effects, and the quality and quantity of interaction (Akçayır and Akçayır, 2017; Altinpulluk, 2019). MARLS enable authentic, interactive, and learner-centred learning experiences (Bacca et al., 2014), deeper knowledge acquisition, and better application of acquired knowledge in different contexts (Radu, 2014).

Despite the potential scalability and the considerable positive effects, MAR-tools are not regularly used in classrooms (Wang et al., 2018). A possible reason for the scarce practical use might be that many teachers still feel insecure when it comes to developing or applying digital learning settings in classroom (Fraillon et al., 2019; Seufert et al., 2019). Although several design principles for creating MAR-tools exist, a lack of principles illustrating how MAR-tools can be effectively used to design complete learning settings has been noted in scholarly discourse (Kerr and Lawson, 2020; Kourouthanassis et al., 2015). While little 
MAR-research has been conducted for the primary and tertiary levels of education, there are even less concepts and empirical data for the secondary level of education (Hedberg et al., 2018).

Consequently, a MARLS is designed that enables teachers to foster Artificial Intelligence (AI)-literacy in upper secondary practice and contribute to the research on design principles of easy-to-use MARLS. AI-literacy, the competence to use smart machines effectively and consciously (Davenport and Kirby, 2016; Long and Magerko, 2020), serves as an exemplary topic. It appears to be suiting because, even if there is consensus that the effective and conscious handling of AI should be fostered at school (Pedro et al., 2019; UNESCO, 2019), in educational practice AI-literacy is still insufficiently addressed (Holmes et al., 2019; Luckin et al., 2016). Additionally, MAR-technology is based on AI-systems such as image recognition (Sahu et al., 2021) and hence allows students to experience a positive form of human-AI interaction whilst using the application.

Two project goals are pursued: 1) the development of a MARLS that enables teachers to foster AI-literacy in practice; 2) the contribution to the scientific discourse on the effective design of easy-to-use MARLS. The Main research Question derives from these goals:

\section{How to design an easy-to-use mobile augmented reality learning setting (MARLS) for upper secondary education by means of conjecture mapping?}

The content of the paper is structured as follows: the next section elaborates why the conjecture map is a favourable tool to design the MARLS and explains the applied educational design research method; the third section provides an overview of the findings: 1) the MARLS design principles 2) the conjecture map and the created learning design; and the last section concludes the paper by discussing the results and providing recommendations for future research.

\section{METHOD}

Design Research allows to pursue the two goals at hand: the development of innovative practical solutions and the advance of theoretical insight on how to do so (Dunning, 2011; Seufert, 2014). The applied educational design research (EDR) developed by McKenney and Reeves, consists of three phases that are conducted iteratively (McKenney and Reeves, 2018; Wozniak, 2015):

1. Analysis and exploration (phase 1): In order to be able to plan and design suitable measures for teachers, the required skills initially have to be discussed and formulated (Sandoval, 2014). The most extensive literature review to conceptualize the competences related to AI-literacy was conducted by Long and Magerko (2020). Their four dimensions "What is AI?", "What can AI do?", "How does AI work?", and "How should AI be used?" and the 16 related competences are therefore applied in the outlined EDR-project. Furthermore, a systematic literature analysis on the construction and application of MAR-tools for educational purpose is conducted to condense the scientific findings to MARLS-design principles. The results of the literature analysis and development of the design principles are presented in detail in (Moser, 2020). However, the most important results are outlined in this contribution to clarify the design principles that build the foundation of the designed AI-literacy MARLS.

2. Design and construction of the learning setting (phase 2): To develop the MARLS, conjecture mapping is used. Conjecture mapping is a proven method to design digital learning settings (Wozniak, 2015). It forces the designers to explicate the main set of assumed relationships (conjectures) and thereby helps to render the applied rationale transparent. The main advantage of the conjecture map is that it puts the focus on the different levels of conjectures that require studying (Sandoval, 2014; Wozniak, 2015) and hence guides phase 3 of the EDR. The conjecture map illustrates three different types of conjectures of the created learning design. The high level conjectures (1) are the basic assumptions - concept of learning goals and understanding of learning and teaching processes. Derived from the high level conjectures, the materials, tasks, and participant structure - the embodiment - is developed. Design conjectures (2) reveal how the embodiment is supposed to induce the observable mediating processes - student artefacts and interactions - which should help to achieve the learning goals. The theoretical conjectures (3) illustrate how the mediating processes are supposed to lead to the desired outcomes (Sandoval, 2016). The research of teaching and learning as isolated processes cannot grasp the complexity of the subject (Deng et al., 2019). 
The Conjecture map allows to conduct research that includes the learning and the teaching dimension and its interdependence in a given learning design (Prensky, 2011) and is therefore a valuable tool for the purpose at hand. The second phase of the EDR process constitutes the main part of this contribution.

3. Evaluation and reflection (phase 3): The conjectures, have to be evaluated and, if necessary, to be adjusted in order to increase the maturity of the design knowledge (Gregor and Hevner, 2013). Additionally, the evaluation of the conjectures allows to improve the MARLS-design. A mixed method-approach will be applied to study what factors contribute to the acceptance and easiness to use a MARLS. To capture the acceptance and willingness of students to use MARLS, a questionnaire that follows the unified theory of technology acceptance and use (Venkatesh et al., 2012) is deployed. Qualitative data contextualizing and enhancing the evaluation is captured in the same survey, to make up for the limited number of participants. In addition, user data from the platform will be included in the analysis. This paper does not address the third step of the EDR process, for the necessary data has yet to be collected. In October 2021 the data of the first 100 students that will have worked with the AI-literacy MARLS, should be available. This will allow to refine the MARLS, the conjectures, and thereby enhance the theoretical understanding of MARLS-development.

\section{RESULTS}

\subsection{Identifying MARLS design principles (phase 1)}

The literature review, presented in Moser (2020), focused on the pedagogical and technical aspects of MAR for education. 77 articles entered the analysis but only twelve studies proposed design principles or frameworks for the construction or application of MAR-tools for educational purposes (Table 1) that also consider pedagogical aspects. The 54 proposed design principles were clustered to five design categories that summarize the main pedagogical and technical challenges when designing easy-to-use MARLS (Figure 1).

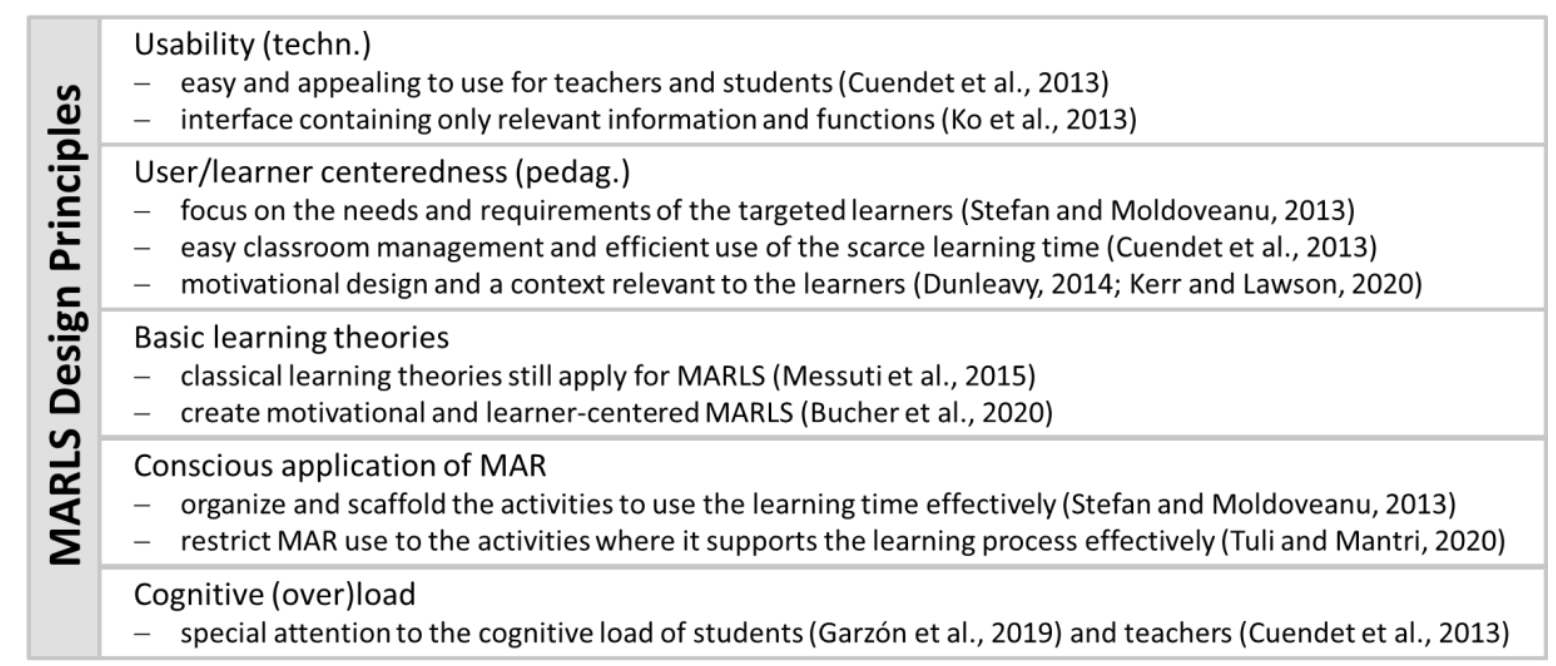

Figure 1. MARLS Design Principles. Moser, 2020

Fundamentally, it seems to be important to design learning settings that fulfil the needs of students and teachers alike. By not only respecting technological design principles and basic learning theories but also including possibilities for a lean classroom-management it seems possible to reduce the cognitive load of both students and teachers (Cuendet et al., 2013). 


\subsection{Design and Conjecture Map of the AI-literacy MARLS (Phase 2)}

To ensure a save digital learning environment, the Xtend platform and the associated application Xpanda (https://www.augmentedreality.ch) will be used for implementing the MARLS. The company has successfully supported several educational projects in Switzerland and guarantees data protection. Furthermore, the mobile application supports iOS and Android and can be downloaded by the students for free. A registration or identification in the application is not necessary. The developed MARLS and the rationale behind it, is outlined along the conjecture map, displayed in Figure 2.

Desired Outcomes: The desired outcome of the learning setting regarding the students equals their learning in the realm of AI-literacy as described by Long and Magerko (2020). The effectiveness of the MARLS regarding the learning effect is a prerequisite for the teachers' acceptance and further use, as it effects the perceived usefulness that has repeatedly shown to be relevant for technology acceptance (Venkatesh et al., 2012). However, the focus of this research project lies on the question of how to design a MARLS that is easy-to-use and accepted by students and teachers alike. Therefore, an objective measurement of AI-literacy is not part of the MARLS.

High level conjectures: Combined with the applied notion of AI-literacy (I.) (Long and Magerko, 2020) and the awareness that a digital learning environment (II.) is necessary, the five design principles outlined in chapter 3.1 embody the high-level-conjectures that guide the development of the MARLS. To ensure a decent usability (III.) and reduce the cognitive load (VII.) of students and teachers alike, the easy-to-use application Xpanda will be used for implementing a marker-based MARLS. User-centeredness (IV.) regarding the teachers' needs is further ensured by developing a complete learning setting that includes a slide set and a booklet entailing all the necessary information to use the MARLS in class. User-centeredness (IV.) regarding the students and conscious application (V.) is implemented through an inquiry-learning approach (Pedaste et al., 2015) where students work in groups of 3-4 on a AI-Map. Marker-based-MAR has shown positive effects in combination with inquiry- or exploration-based learning (VI.) in multiple studies (Billinghurst and Duenser, 2012; Bower et al., 2015; Puja and Parsons, 2011). Also, MAR-enhanced learning appears to be valuable for intensifying interaction with the learning material and between members of learning groups (Akçayır and Akçayır, 2017; Altinpulluk, 2019).

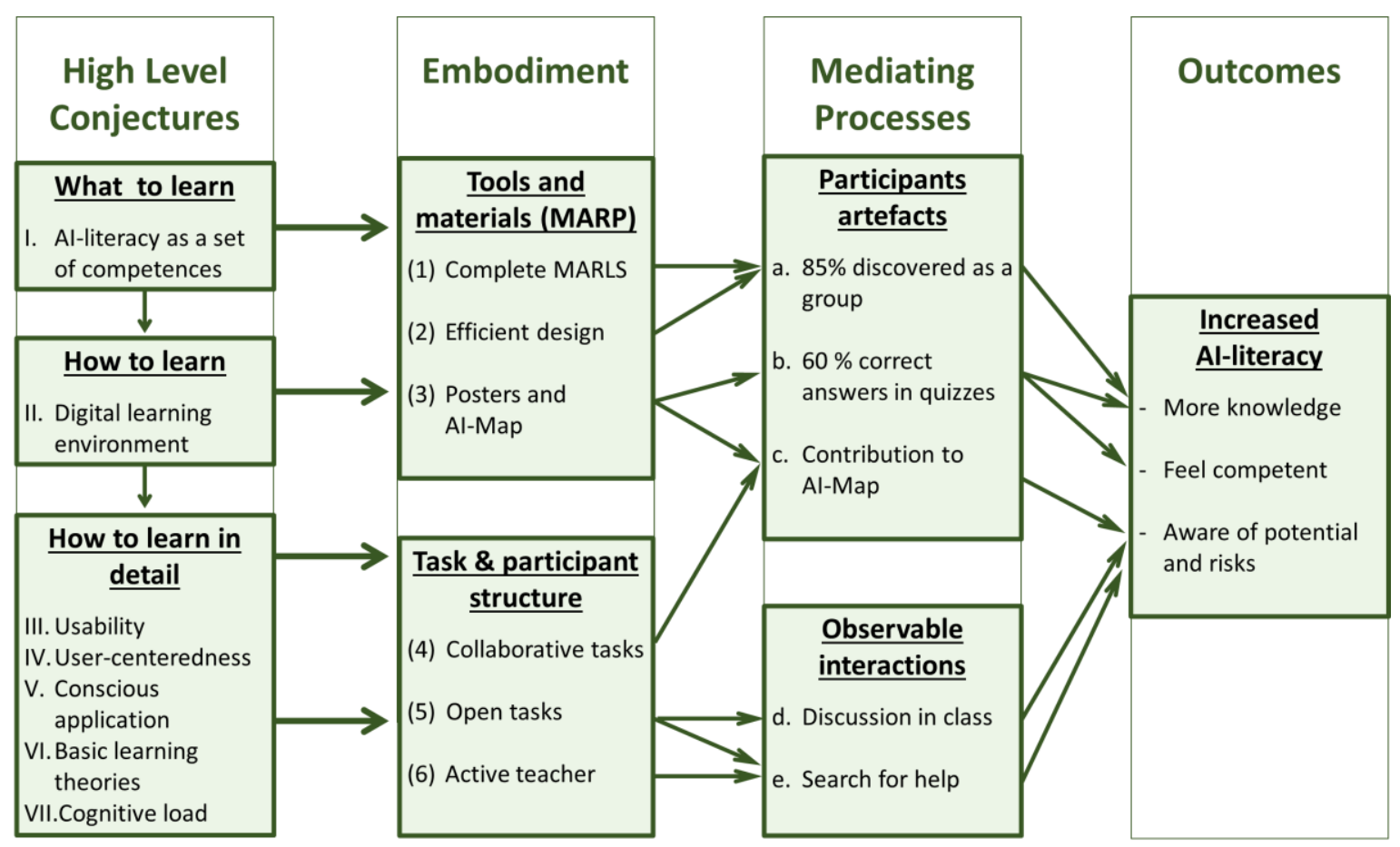

Figure 2. MARLS Conjecture Map 
Embodiment: Because of the teachers' insecurities (Fraillon et al., 2019) a complete MARLS (1) is provided and additional material for an easy implementation in class is provided. To ensure an efficient design (2), after a brief introduction to AI and the MAR-app, the students start to work with the posters to complete their AI-Map (3). In addition to basic theories and applications about smart machines and AI, they explore which AI-systems are necessary for social robot 'Lexi' to work properly. To enable the students to learn in an inquiry- and exploration-based setting, open and collaborative tasks (4)(5) are formulated. When scanning the picture-based markers on the AI-Map and the posters (see Figure 3), students activate quizzes, videos, readings and visualizations experiencing the underlying relationships in the field of smart machines, to enhance their AI-literacy. In groups of 3-4, the students enhance their knowledge and awareness about smart machine applications - e.g. the topic of natural language processing and translation is explored by actively deploying "DeepL" and "Google Translate". Earlier projects with digital learning settings (Moser et al., 2020; Moser et al., 2021) indicate that students and teachers might both appreciate and profit from an active involvement (6) and support of the teacher in the students' digitally supported learning process.

Mediating processes: Students collect the physical markers provided as stickers on the posters and contribute to the groups AI-Map (c.) by discussing (d.) the potentials and possible dangers of the respective applications, they should engage intensively with the learning materials. By building basic knowledge and gaining practical insights, the students are enabled to build a reflected opinion and shape their attitudes (Ingram, 2015; Zan and Di Martino, 2007). Furthermore, the Xpanda platform allows the teacher to monitor the students' activity and interaction with the learning materials and thereby to monitor the rate of discovery (a.) and the rate of correct responses (b.). This information facilitates the teacher to take an active role and support the learning processes of the students when they are looking for help (e.) or need help but do not approach the teacher on their own.

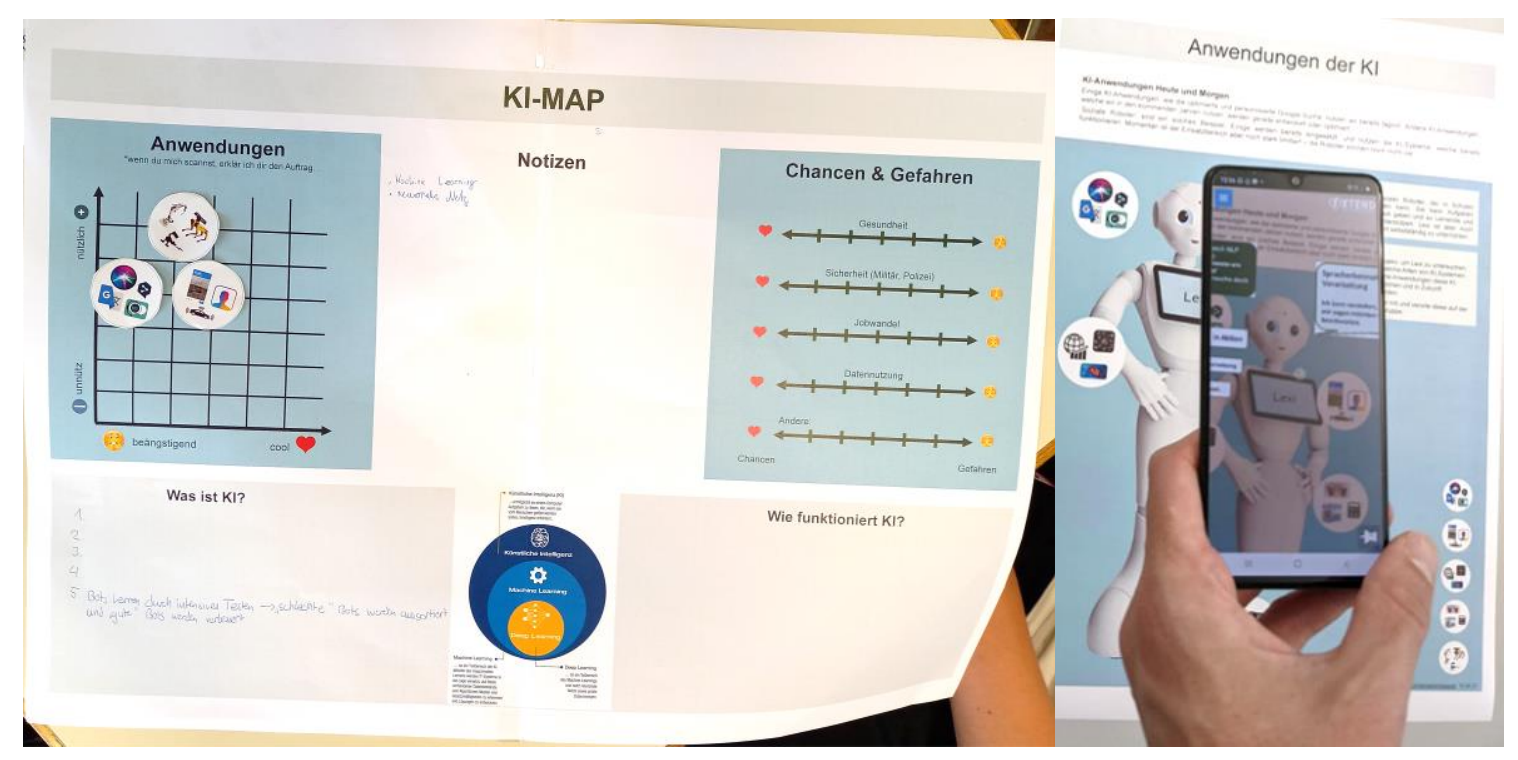

Figure 3. AI-Map and Lexi-poster with AI-supported applications

\section{CONCLUSION}

Limitations: The outlined EDR-cycle is still in its first iteration. The MARLS-evaluation is yet to follow. Furthermore, in order to increase the overarching validity, compromises in terms of reliability and validity of isolated effects are made and have to be respected when interpreting and discussing the results. Previous projects studying digitally enhanced learning processes (Moser et al., 2020; Moser et al., 2021) indicate that considerable novelty-effects are a further challenge for the validity of studies regarding innovative learning settings. Only the repeated use of MARLS really allows to control for these novelty effects. Further investigations have to clarify which findings can be transferred to other MARLS and other educational levels. 
Another important limitation is the fast evolvement of MAR-technology (Cuendet et al., 2013) and the increasing capacities of the smartphones the average students possess and use to learn with MARLS. The technical possibilities are likely to change, how a conscious application (Tuli and Mantri, 2020) can be achieved. Despite these numerous limitations, the present project may contribute to the further exploration on how to facilitate the transfer of scientific innovation into practical learning environments.

Theoretical and Practical Implications: The MARLS is designed as an open educational resource and can therefore be used in classrooms by any interested teacher and student. Feedbacks from and interviews with further participants may produce more theoretical insight and practical improvement of the work with MARLS in the second and third EDR-cycle. The need to empirically study the perceptions and competence levels of students, concerning AI-literacy, identified by Long and Magerko (2020) could also be addressed in future research using the developed AI-MARLS.

For the practical application and dissemination of MARLS, the availability of simple development tools will probably be a relevant factor. Considering the amount of new digital learning solutions and approaches that are developed (Brooks et al., 2020), it appears crucial to intensify the scientific discourse about how to allow educational innovations to enter the classrooms more easily. The ongoing research on MARLS might contribute to do so.

\section{REFERENCES}

Akçayır, M. and Akçayır, G., 2017. Advantages and challenges associated with augmented reality for education: A systematic review of the literature. Educational Research Review, Vol. 20, pp. 1-11.

Altinpulluk, H., 2019. Determining the trends of using augmented reality in education between 2006-2016. Education and Information Technologies, Vol. 24 No. 2, pp. 1089-1114.

Azuma, R.T., 1997. A survey of augmented reality. Presence: Teleoperators \& Virtual Environments, Vol. 6 No. 4 , pp. 355-385.

Bacca, J., et al., 2014. Augmented Reality Trends in Education: A Systematic Review of Research and Applications. Educational Technology \& Society, Vol. 17 No. 4, pp. 133-149.

Billinghurst, M., and Duenser, A., 2012. Augmented reality in the classroom. Computer, Vol. 4 No. 7, pp. 56-63.

Bower, M., et al., 2014. Augmented Reality in education-cases, places and potentials. Educational Media International, Vol. 51 No. 1, pp. 1-15.

Brooks, D. C., et al., 2020. Higher Education's 2020 trend watch and top 10 strategic technologies. EDUCAUSE. Retrieved 2020/09/01 from: https://library.educause.edu/resources/2020/1/higher-educations-2020-trend-watch-andtop-10-strategic-technologies

Bucher, K., et al., 2020. Von Medienbeiträgen und Applikationen-ein interdisziplinäres Konzept zum Lehren und Lernen mit Augmented und Virtual Reality für die Hochschullehre. Preprint, pp. 225-238.

Cano, E.V. and Sevillano-García, M.L., 2018. Ubiquitous Educational Use of Mobile Digital Devices. A General and Comparative Study in Spanish and Latin America Higher Education. New Approaches Educational Research, Vol. 7, pp. 105-115.

Cuendet, S., et al., 2013. Designing augmented reality for the classroom. Computers \& Education, Vol. 68, pp. 557-569.

Davenport, T. H. and Kirby, J., 2016. Just how smart are smart machines?. MIT Sloan Management Review, Vol. 57 No. 3, pp. 21-25.

Davies, E. R., 2004. Machine vision: theory, algorithms, practicalities, ( $3^{\text {rd }}$ ed.). Academic Press, New York, USA.

Deng, R., et al., 2019. Progress and new directions for teaching and learning in MOOCs. Computers \& Education, Vol. 129, pp. 48-60.

Dunleavy, M., 2014. Design principles for augmented reality learning. TechTrends, Vol. 58 No. 1, pp. 28-34.

Dunning, D., 2011. Chapter five - The Dunning-Kruger Effect: On Being Ignorant of One's Own Ignorance. Advances in Experimental Social Psychology, Vol. 44, pp. 247-296.

Fraillon, J., et al., 2019. Preparing for life in a digital world: the IEA International Computer and Information Literacy Study 2018 International Report. IEA, Amsterdam, Netherlands.

Garzón, J., et al., 2019. Systematic review and meta-analysis of augmented reality in educational settings. Virtual Reality, Vol. 23 No. 4, pp. 447-459. 
Gregor, S. and Hevner, A. R., 2013. Positioning and presenting design science research for maximum impact. MIS quarterly, Vol 39 No. 2, pp. 337-355.

Hedberg, et al., 2018. A Systematic Review of Learning through Mobile Augmented Reality. International Journal of Interactive Mobile Technologies (iJIM), Vol. 12 no. 3, pp. 75-85.

Holmes, W., et al., 2019. Artificial intelligence in education. Center for Curriculum Redesign, Boston, USA.

Ingram, N., 2015. Students' relationships with mathematics: Affect and identity. In M. Marshman, et al. (Eds.), Mathematics education in the margins. Proceedings of the 38th annual conference of the Mathematics Education Research Group of Australasia, MERGA, Sunshine Coast, Australia, pp. 301-308.

Kerr, J. and Lawson, G., 2020. Augmented Reality in Design Education: Landscape Architecture Studies as AR Experience. International Journal of Art \& Design Education, Vol. 39 No. 1, pp. 6-21.

Ko, S.M., Chang, W.S. and Ji, Y.G., 2013. Usability Principles for Augmented Reality Applications in a Smartphone Environment. International Journal of Human-Computer Interaction, Vol. 29 No. 8, pp. 501-515.

Kourouthanassis, P.E., et al., 2015. Demystifying the design of mobile augmented reality applications. Multimedia Tools and Applications, Vol. 74 No. 3, pp. 1045-1066.

Long, D. and Magerko, B., 2020. What is AI literacy? Competencies and design considerations. Proceedings of the 2020 CHI Conference on Human Factors in Computing Systems, April, 2020, pp. 1-16.

Luckin, R., et al., 2016. Intelligence unleashed: An argument for AI in education. Pearson Education, London, UK.

McKenney, S. and Reeves, T. C., 2014. Educational design research. Handbook of research on educational communications and technology. pp. 131-140, Springer, New York, USA.

Messuti, A., et al., 2015. Usability principles to design mobile workplace learning content. Open Journal per la formazione in rete, Vol. 15 No. 1, pp. 118-128.

Milgram, P., and Kishino, F., 1994. A taxonomy of mixed reality visual displays. IEICE TRANSACTIONS on Information and Systems, Vol. 77 No. 12, pp. 1321-1329.

Moser, L., 2020. Mobile Augmented Reality: Design Principles for the Use in Educational Settings. Proceedings of the $17^{\text {th }}$ International Conference on Cognition and Exploratory Learning in Digital Age, CELDA 2020. Lisbon, Portugal, pp. 335-339.

Moser, L., et al., 2020. Lehrer*innenbildung von digitalen Kompetenzen in einer forschungsbasierten Lerngemeinschaft. Fallbeispiel i-MOOC zur Förderung von Informationskompetenzen auf der Sekundarstufe II. In Kaspar, K., et al., (Eds.), Bildung, Schule, Digitalisierung. Waxmann, Münster, Germany, pp. 203-209.

Moser, L., et al., 2021. Improving a MOOC to foster information literacy by means of a conjecture map. International Journal of Learning Technology, Vol. 16 No. 1, pp. 65-86.

Pedaste, M., et al., 2015. Phases of inquiry-based learning: Definitions and the inquiry cycle. Educational research review, Vol. 14, pp. 47-61.

Pedro, F., et al., 2019. Artificial intelligence in education: Challenges and opportunities for sustainable development. UNESCO.

Prensky, M., 2011. Digital natives, digital immigrants Part 1. On the Horizon, Vol. 9 No. 5, pp. 1-6.

Puja, J. C., and Parsons, D., 2011,. A location-based mobile game for business education. 2011 IEEE 11th International Conference on Advanced Learning Technologies, Washington DC, USA, pp. 42-44.

Radu, I., 2012. Why should my students use AR? A comparative review of the educational impacts of augmented-reality. 2012 IEEE International Symposium on Mixed and Augmented Reality (ISMAR), Atlanta, USA, pp. 313-314.

Sahu, C. K., et al., 2021. Artificial intelligence (AI) in augmented reality (AR)-assisted manufacturing applications: A review. International Journal of Production Research, Vol. 59 No. 16, pp. 4903-4959.

Sandoval, W., 2014. Conjecture Mapping: An Approach to Systematic Educational Design Research. Journal of the Learning Sciences, Vol. 23 No. 1, pp. 18-36.

Seufert, S., 2014. Potenziale von Design Research aus der Perspektive der Innovationsforschung. Zeitschrift für Berufs- und Wirtschaftspädagogik, Beiheft Vol. 2 No. 27, pp. 79-96.

Seufert, S., et al., 2019. Professionelle Kompetenzen von Lehrpersonen im Kontext des digitalen Wandels. Zeitschrift für Berufs-und Wirtschaftspädagogik, Vol. 115 No. 2, pp. 312-339.

Stefan, L. and Moldoveanu, F., 2013. Game-based learning with Augmented Reality-from technology's affordances to game design and educational scenarios. The International Scientific Conference eLearning and Software for Education, 2, 105-114.

Suter, L., et al., 2018. JAMES - Jugend, Aktivitäten, Medien - Erhebung Schweiz. Zürcher Hochschule für Angewandte Wissenschaften, Zurich, $\mathrm{CH}$. 
Tuli, N. and Mantri, A., 2020. Usability Principles for Augmented Reality based Kindergarten Applications. Procedia Computer Science, Vol. 172, pp. 679-687.

UNESCO, 2019. beijing consensus on artifcial intelligence and education. Outcome document of the International Conference on Artificial Intelligence and Education 'Planning education in the AI era: Lead the leap', May 2019, Beijing, People's Republic of China.

Venkatesh, V., et al., 2012. Consumer acceptance and use of information technology: extending the unified theory of acceptance and use of technology. MIS quarterly, Vol. 36 No. 1, pp. 157-178.

Wang, M., et al., 2018. Augmented reality in education and training: pedagogical approaches and illustrative case studies. Journal of ambient intelligence and humanized computing, Vol. 9 No. 5, pp. 1391-1402.

Wozniak, H., 2015. Conjecture mapping to optimize the educational design research process. Australasian Journal of Educational Technology, Vol. 31 No. 5, pp. 597-612.

Zan, R. and Di Martino, P., 2007. Attitude toward mathematics: Overcoming the positive/negative dichotomy. The Montana Mathematics Enthusiast, Vol. 3 No. 1, pp. 157-168. 